\title{
A deep, wide-field search for substellar members in NGC 2264
}

\author{
T. R. Kendall ${ }^{1}$, J. Bouvier ${ }^{1}$, E. Moraux ${ }^{2}$, D. J. James ${ }^{1,3}$, and F. Ménard ${ }^{1}$ \\ ${ }^{1}$ Laboratoire d'Astrophysique, Observatoire de Grenoble, Université Joseph Fourier, 38041 Grenoble Cedex 09, France \\ e-mail: [tkendall; jbouvier]@obs.ujf-grenoble.fr \\ 2 Institute of Astronomy, University of Cambridge, Madingley Road, Cambridge CB3 OHA, UK \\ e-mail:moraux@ast.cam.ac.uk \\ 3 Department of Physics and Astronomy, Vanderbilt University, 1807 Station B, Nashville, TN 37235, USA*
}

Received 12 October 2004 / Accepted 16 January 2005

\begin{abstract}
We report the first results of our ongoing campaign to discover the first brown dwarfs (BD) in NGC 2264, a young ( $3 \mathrm{Myr}$ ), populous star forming region for which our optical studies have revealed a very high density of potential candidates -236 in $<1 \mathrm{deg}^{2}-$ from the substellar limit down to at least $\sim 20 M_{\text {Jup }}$ for zero reddening. Candidate BD were first selected using wide field $(I, z)$ band imaging with CFH12K, by reference to current theoretical isochrones. Subsequently, 79 (33\%) of the $I, z$ sample were found to have near-infrared 2MASS photometry $\left(J H K_{\mathrm{s}} \pm 0.3 \mathrm{mag}\right.$ or better), yielding dereddened magnitudes and allowing further investigation by comparison with the location of NextGen and DUSTY isochrones in colourcolour and colour-magnitude diagrams involving various combinations of $I, J, H$ and $K_{\mathrm{s}}$. We discuss the status and potential substellarity of a number of relatively unreddened $\left(A_{\mathrm{v}} \lesssim 5\right)$ likely low-mass members in our sample, but in spite of the depth of our observations in $I, z$, we are as yet unable to unambiguously identify substellar candidates using only 2MASS data. Nevertheless, there are excellent arguments for considering two faint (observed $I \sim 18.4$ and 21.2) objects as cluster candidates with masses respectively at or rather below the hydrogen burning limit. More current candidates could be proven to be cluster members with masses around $0.1 M_{\odot}$ via gravity-sensitive spectroscopy, and deeper near-infrared imaging will surely reveal a hitherto unknown population of young brown dwarfs in this region, accessible to the next generation of deep near-infrared surveys.
\end{abstract}

Key words. stars: low-mass, brown dwarfs - infrared: stars - surveys - Galaxy: open clusters and associations: individual: NGC 2264

\section{Introduction}

Within the last few years the observational study of substellar objects $\left(M<0.072 M_{\odot}\right)$ has undergone spectacular and rapid development, revealing new perspectives on the formation of such objects within molecular clouds. Large numbers of brown dwarfs (BD) have now been found in star-forming regions (SFRs), young clusters and the field (Béjar et al. 2001; Moraux et al. 2003; Chabrier 2002, 2003; Cruz et al. 2003) and physical models of the atmospheres of BD constructed (Baraffe et al. 2003; Chabrier et al. 2000), opening up the possibility of confrontation between observations and theoretical predictions of the physical properties of BD. However, the core issues of the form of the substellar initial mass function (IMF) and its dependence on environment remain to be addressed. The discovery of BD in widely differing environments does suggest their formation is directly linked to the star formation process, and early estimates of the substellar IMF in the solar neighbourhood have indicated

^ Based on observations made with the Canada-France-Hawaii telescope and data from the 2MASS project (University of Massachusetts and IPAC/Caltech, USA). that BD are nearly as numerous as stars. Within this broad framework, two competing scenarios have been put forward for BD formation; the first simply that they form as stars do, i.e. by the gravitational collapse of low mass molecular cloud cores (Padoan \& Nordlund 2002); the second postulates the dynamical ejection of the lowest mass protostars, leading to BD formation since the ejected fragments are unable to further accrete (Reipurth \& Clarke 2001). To distinguish between these possibilities and to obtain unbiased estimates of the substellar IMF, observations of statistically complete, homogeneous populations of BD are needed in a wide variety of environments with different ages.

Young star-forming regions are of particular interest since the youth of such objects ensures that their population has not yet suffered from important dynamical (and stellar) evolution, other than dynamical effects potentially associated with their formation. This is a point worth underlining, since observations of young SFRs have the potential to distinguish these two BD formation mechanisms by their resultant spatial distributions. If $\mathrm{BD}$ always form exactly as stars do, one would expect them to trace the regions of the highest density of low-mass stars. However, in any dynamical ejection scenario, 
a deficit of BD may be expected in the central regions of young star-forming clusters. Their initial velocities of a few $\mathrm{km} \mathrm{s}^{-1}$ (Sterzik \& Durisen 2003) would especially imply a preferential loss of BD from loosely bound regions such as Taurus, where a velocity of $1 \mathrm{~km} \mathrm{~s}^{-1}$ would correspond to a drift of $\sim 0.3^{\circ} / \mathrm{Myr}$ at $140 \mathrm{pc}$ (or in absolute terms $\sim 0.7 \mathrm{pc} / \mathrm{Myr}$ ). In denser environments, BD would remain bound, but Moraux \& Clarke (2005) found that BD would still be expected to have a more extended distribution than the stars, in clusters whose age is comparable to the crossing time. Such a difference in spatial distribution should be observable in all nearby such SFRs; we note also that other models predict rather similar dispersion velocities for BD and more massive members (Bate et al. 2003). Wide field observations of large BD samples in these regions will soon settle this issue.

Young BD (with ages 1-3 Myr) are now being uncovered (for a relatively recent compilation see Basri 2000), but the census remains very incomplete to date. Comparison between the BD populations of young SFRs with a range of environmental conditions permits investigation of the sensitivity of the low-mass end of the IMF to local conditions, and other more recent studies are beginning to address this question. To cite two specific cases, 30 substellar candidates have been identified in Taurus in a $3.6 \mathrm{deg}^{2}$ region using CFH12K data which reached to $I=23.5$. Spectroscopic follow-up led to the identification of four BD in Taurus with spectral type later than M 7 (Martín et al. 2001). In Taurus, 10 BD are now known (Briceño et al. 2002), and appear to be spatially correlated with regions of highest stellar density. In Upper Scorpius, initial studies (Martín et al. 2004) have found 18 candidate BD of which 5 show signs of ongoing accretion.

In this paper, we present initial results from a deep CFH12K survey of the young ( $3 \mathrm{Myr}$ ), rich star-forming region NGC 2264. The study introduced here is only a small part $\left(0.6 \mathrm{deg}^{2}\right)$ of a much broader survey, which has mapped significant areas $\left(70 \mathrm{deg}^{2}\right)$ of a variety of environments (ages 1-600 Myr) sufficiently deeply to probe BD; in all of the targeted regions, the limiting mass is in the range 10-40 $\mathrm{M}_{\mathrm{Jup}}$. NGC 2264, because of its youth and relative proximity (3 Myr, 760 pc; Lamm et al. 2004; Rebull et al. 2002; Sung et al. 1997; Park et al. 2000), is probed to the lower end of this mass range with the current observations. We will discuss our initial findings on NGC 2264 in Sect. 4; here we will note that the much larger coeval BD populations currently being uncovered by CFH12K, when fully analysed, promise a more robust statistical treatment of both the kinematics of BD populations and variations in the substellar IMF from region to region.

\section{Observations of candidate NGC 2264 members: Optical colour selection}

Observations of NGC 2264 were carried out using the CanadaFrance-Hawaii Telescope (CFHT) and the $12 \mathrm{~K}$ camera, with a mosaic of $124128 \times 208015 \mu \mathrm{m}$ pixel CCDs as detector yielding a field of view of $43^{\prime} \times 28^{\prime}$ with $0.206^{\prime \prime}$ pix $^{-1} .2$ fields were observed in the $I$-band on 2 February 1999, and the same fields covered in the $z$-band on 19 December 2000; in both cases each observation is a composite of $3 \times 360 \mathrm{~s}$ exposures. Weather conditions were good and the seeing 1.2" or better. The pre-reduction and analysis of these data have been performed using CFHT Elixir ${ }^{1}$ pipelines and new, innovative point-spread function (PSF) fitting techniques (SExtractor, PSFex) developed at the Institut d'Astrophysique de Paris by E. Bertin (Bertin \& Amouts 1996) ${ }^{2}$. After pipeline image flatfielding and co-addition, $z$-band images (taken at a later epoch) were mapped on to the $I$-band images using standard techniques (GEOMAP, GEOTRANS) within the IRAF $^{3}$ environment. Photometric extraction was initially performed on the $I$-band images, with subsequent matching to the $z$-images with an accuracy of \pm 5 pixels. The assessment of photometric zeropoints at $I$ was provided using images of the Landolt standard field SA 101 (Landolt 1992), observed with the same instrumental setup at the same epoch. Where there were sufficient standard stars in each individual CCD of the array (around half the $12 \mathrm{CCDs}$ ) CCD-to-CCD variations in the zeropoint could be estimated, compared with the Elixir pipeline values (no discrepancies larger than 0.1-0.2 mag were found) and taken into account in our analysis. For the other I-band observations, zeropoints were taken directly from the night-by-night CFHT/Elixir database; this was also the case for the later-epoch $z$-band observations, which are calibrated assuming $I-z=0$ for A0 stars. We estimate our typical random photometric errors to be $\pm 0.1 \mathrm{mag}$ at $I=23$ and $\pm 0.05 \mathrm{mag}$ at $I=22$, allowing us to place typical error bars in Fig. 1 which pertain near the completeness limit.

We performed a number of sample refining and cleaning procedures as follows; firstly; photometric points found to have undefined magnitudes following PSF-fitting were rejected; this accounts for many detections close to bad columns, in diffraction spikes, etc. Candidates flagged to be saturated and/or near field edges were then also removed. At this point, the statistics of the remaining detections, placed in one-magnitude bins in $I$, suggested that the data should be complete to very nearly $I=23$, there being very similar numbers of objects in this faintest bin and one magnitude brighter. Subsequently, we retained only objects with a fraction-of-light radius ("flux radius") within quite narrow ranges estimated by eye by looking at plots of this quantity versus magnitude. For example, in $I$ only objects with a value of between 2 and 3.5 pixels were retained. Furthermore, we rejected objects with an ellipticity of greater than 0.15 , in a very efficient attempt to rid our sample of extended or non-stellar objects. (To illustrate this, only one of 236 visually inspected candidates failed the ellipticity cut; it is clear that this cut is an efficient way of refining the sample, avoiding individual inspection of spurious points.) As a final check on our selection, all 236 such $I, z$ candidates (squares in Fig. 1) were carefully inspected visually on both $I$ and $z$ frames and all found to be stellar in nature, i.e. there are no artifacts due to nebulosity, field edges, bad columns, bright stars etc.

\footnotetext{
1 www.cfht.hawaii.edu

2 See www.terapix.iap. fr for more recent developments.

${ }^{3}$ IRAF is distributed by the National Optical Astronomy Observatories, which is operated by the Association of Universities for Research in Astronomy, Inc. (AURA) under cooperative agreement with the National Science Foundation.
} 


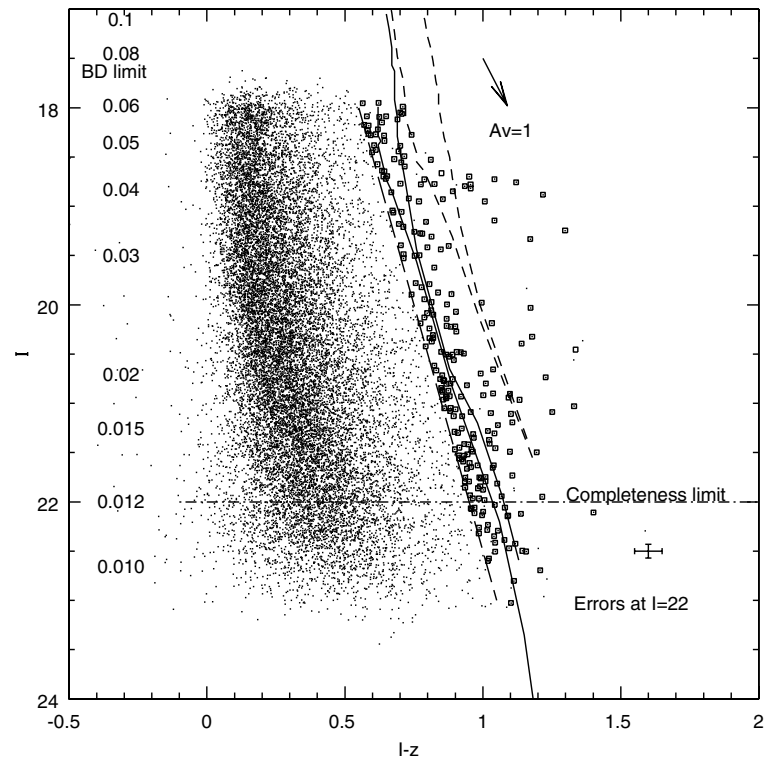

Fig. 1. $I, I-z$ colour-magnitude diagram built using long $(3 \times 360 \mathrm{~s})$ exposures of NGC 2264. The solid isochrones are DUSTY models for ages of 2 and $5 \mathrm{Myr}$; the dashed isochrones are the NextGen models for the same ages. All isochrones are for distance modulus $9.4 ; 760 \mathrm{pc}$. 236 BD candidates (squares) were selected to be redward of the sloping straight line (long dash). Small dots redward of this line were rejected from the candidacy on visual inspection. Typical error bars for $I=22$ of $\sim 0.1 \mathrm{mag}$ are shown. The mass scale (in $M_{\odot}$ ) is for the $2 \mathrm{Myr}$ models; it and the estimated completeness limit are indicated.

After all these steps, we retained all 236 candidates but only plot field objects satisfying $e<0.15$, and all other criteria, in Fig. 1. (Hence, the one candidate failing this criterion is the only point in Fig. 1 to be plotted as an open square with no inner dot.) We find that the number of objects with $I=22-23$ is roughly $50 \%$ of the number in the $I=21-22$ range; hence a completeness limit in the refined sample of $I \sim 22$ is reasonable. We note that we made no attempt to remove candidates on the basis of their magnitudes only; our cuts have rejected no fainter candidates, but a much larger number of points which prove, for one reason or another, clearly spurious on inspection. Finally, astrometric calibration was carried out for each individual candidate using the Starlink ASTROM package. With an initial selection of $\sim 10$ stars within the same CCD as the candidate, and input positions taken from the USNO A 2.0 catalogue, we found RMS accuracies of the initial fits to better than $0.1^{\prime \prime}$.

Figure 1 shows optically selected candidates in NGC 2264 between the substellar limit and $\sim 10 M_{\mathrm{Jup}}$, by comparison with the state-of-the-art DUSTY models, specially created by Baraffe et al. to take account of the CFHT $I, z$ filter responses (see Baraffe et al. 2003, and references therein for full details of these models). In order to account for uncertainties in the model loci, and remain conservative in our candidate selection, all objects redward of the sloping dashed line in Fig. 1 were retained for further investigation. As can also be seen in Fig. 1, our estimate of completeness to $I \sim 22$, or $12 M_{\text {Jup }}$ for age 2 Myr (unreddened), appears accurate.

\section{Dereddening the sample}

In this section, we discuss our methods to estimate the extiction towards each member of our candidate sample. A brief examination of the reddening derived by previous authors is relevant at this point. At the galactic coordinates for NGC 2264 given by Rebull et al. (2002), $(l=202.96, b=+2.22)$ the maps of Schlegel et al. (1998) yield $A_{\mathrm{v}}=5.63$, but it is considered that a background dark molecular cloud exists (Rebull et al. 2002), limiting contamination by background giants. Indeed, these authors find a typical extinction towards NGC 2264 members $A_{\mathrm{v}} \sim 0.45$ (from photometry and spectroscopy of latetype members), in good agreement with earlier values derived using small numbers of OB stars (Sung et al. 1997; Park et al. 2000). Noting that the foreground reddening is effectively zero (Sung et al. 1997), we therefore assume that $A_{\mathrm{v}}$ toward any given object in our sample may vary from zero to high values. Futhermore, with such large and variable extinction intrinsic to NGC 2264, it is clear that there may be relatively unreddened objects (perhaps on the nearer side of the cloud), and more deeply embedded objects.

We find variable extinction values ranging up to $A_{\mathrm{v}} \sim 15$, but generally in the range $0-10$. Our methods rely on the recovery of 79 objects in our sample of 236 (33\%) in the 2MASS database ${ }^{4}$, where 2 MASS detections were required to be within $2^{\prime \prime}$ of our optical positions. More 2MASS counterparts exist, but with either $J$ - or $K_{\mathrm{s}}$-magnitudes only given as upper limits - we do not consider these objects further. The 79 objects will form the basis of the current study; all have photometric flags $\mathrm{A}, \mathrm{B}$ or $\mathrm{C}$, corresponding to errors $\pm 0.1 \mathrm{mag}(\mathrm{A})$ to $\pm 0.3 \mathrm{mag}(\mathrm{C})$.

The basis for dereddening is given in Figs. 2 and 3. We have used observed loci for late-type dwarfs from compilations given by Leggett (1992), Leggett et al. (1998), Bessell (1991) and Kirkpatrick et al. (2000) to compare the location of our sample in the $I-J, J-K_{\mathrm{s}}$ and $J-H, H-K_{\mathrm{s}}$ colour-colour diagrams. In order to verify our methods we first checked our sample for obvious $K_{\mathrm{s}}$-band excesses, which would strongly affect estimates of the extinction which used the $K_{\mathrm{s}}$-magnitude. We found none (Fig. 4).

We plotted the location of our sample in $I-J, J-K_{\mathrm{s}}$, in which diagram the location of an unreddened $M$-dwarf locus can be easily described by a straight line (Fig. 2). From the known slope of the reddening vector (Rieke \& Lebofsky 1985) and the gradient of the line (dotted in Fig. 2) the magnitude of the vector $A_{\mathrm{v}}$ can be calculated via simple geometric arguments. Exactly similar arguments can be applied in the case of Fig. 3, where only near-infrared colours are utilised. In this case, the photometric errors are larger, with respect to the range of $J H K_{\mathrm{s}}$ colours observed. Hence, in Fig. 3, we make a clear distinction between those objects (42 out of 79) which have precise 2MASS photometry and those for which the errors are likely larger than \pm 0.1 mag We find that the values of $A_{\mathrm{v}}$ found by these two methods are almost identical, aside from a few objects for which $A_{\mathrm{v}}$ is low and the difference between the value of the extinction given by the two methods is of order $\sim 1$ mag

\footnotetext{
4 www.ipac.caltech/edu/2MASS
} 


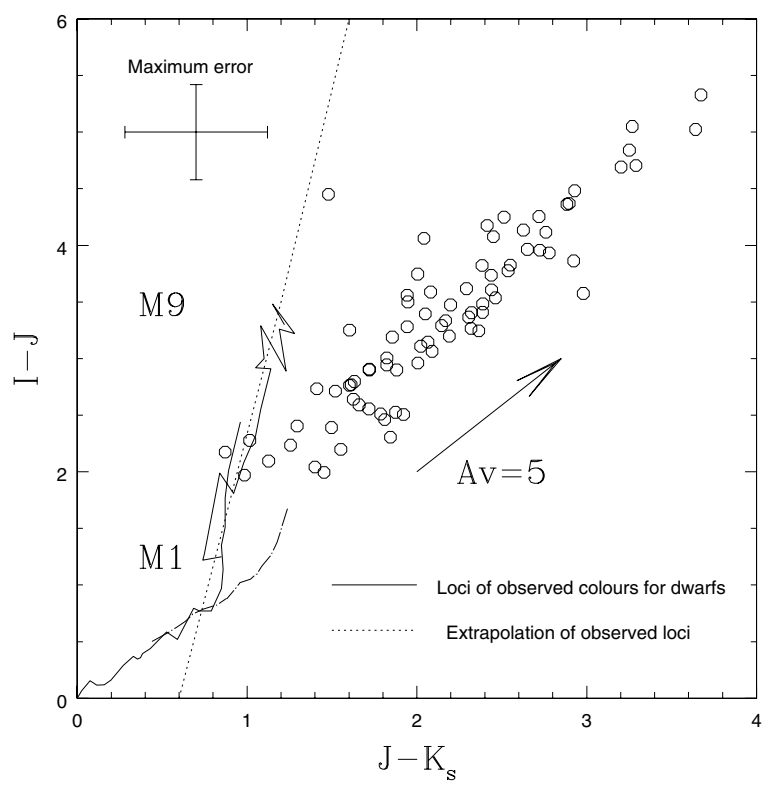

Fig. 2. All $79 I z J H K_{\mathrm{s}}$ candidates (open circles), plotted in the $I-J$, $J-K_{\mathrm{s}}$ diagram. The errorbar assumes $J H K_{\mathrm{s}}$ accurate to $\pm 0.3 \mathrm{mag}$ and $I$ to $\pm 0.1 \mathrm{mag}$ The reddening vector is shown. The solid lines are observed loci of dwarfs taken from a compilation of sources (see text). The dash-dot line is a the locus of G0-M 5 giants from Bessell \& Brett (1988). $A_{\mathrm{v}}$ has been computed by dereddening all points to the extrapolated (dotted) locus of dwarfs. After dereddening, all objects are potentially either cluster or field $M$-dwarfs.

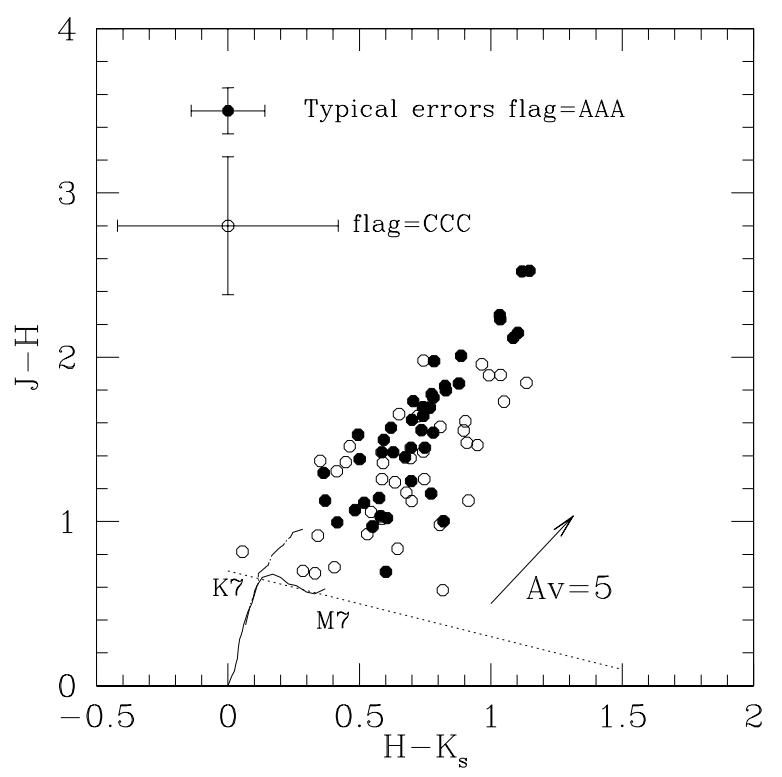

Fig. 3. Similar to Fig. 2, but for $J H K_{\mathrm{s}}$. Since the colours plotted here vary relatively less among the sample, the errorbars appear larger. Therefore, a distinction has been made between the best 2MASS photometry (all magnitudes flagged A, filled points) and those with any magnitude flagged $\mathrm{B}$ or $\mathrm{C}$. For the former, $J H K_{\mathrm{s}}$ are taken accurate to $\pm 0.1 \mathrm{mag}$, for the latter, $\pm 0.3 \mathrm{mag}$, hence the errorbars plotted. Again, $A_{\mathrm{v}}$ has been calculated by dereddening to the extrapolated (dotted) locus of observed late-type dwarf colours. The locus of giant colours is also plotted (dash-dot line).

(Fig. 5), which is not a significant difference in the context of this work. Hence, in order to retain the important I-band

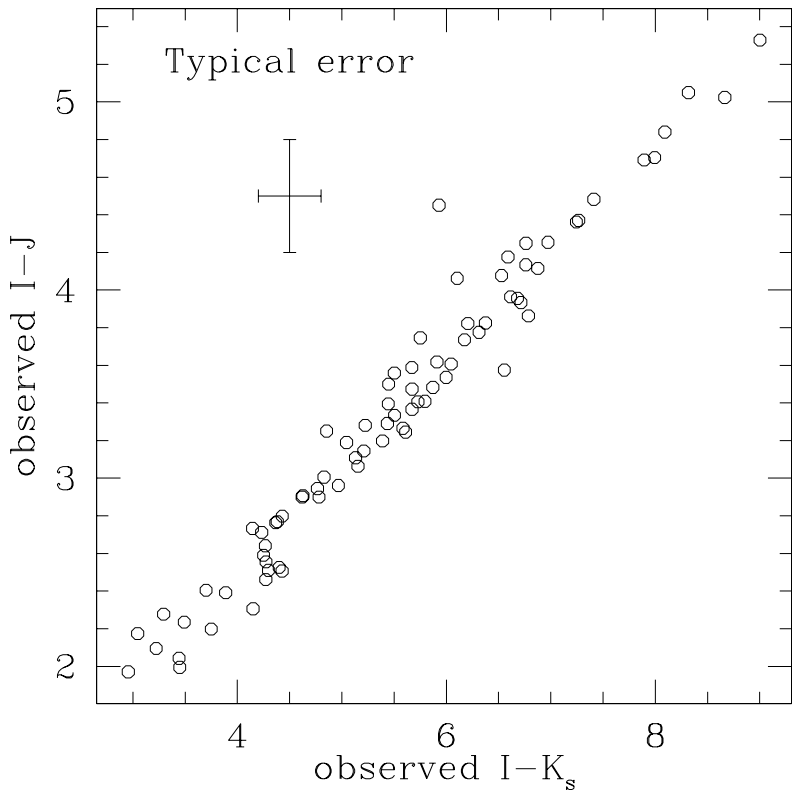

Fig. 4. To check for obvious $K_{\mathrm{s}}$-band excesses which may affect our derivation of $A_{\mathrm{v}}$, we have plotted all 79 candidates in the $I-J, I-K_{\mathrm{s}}$ diagram. Clearly, no objects have significant excesses.

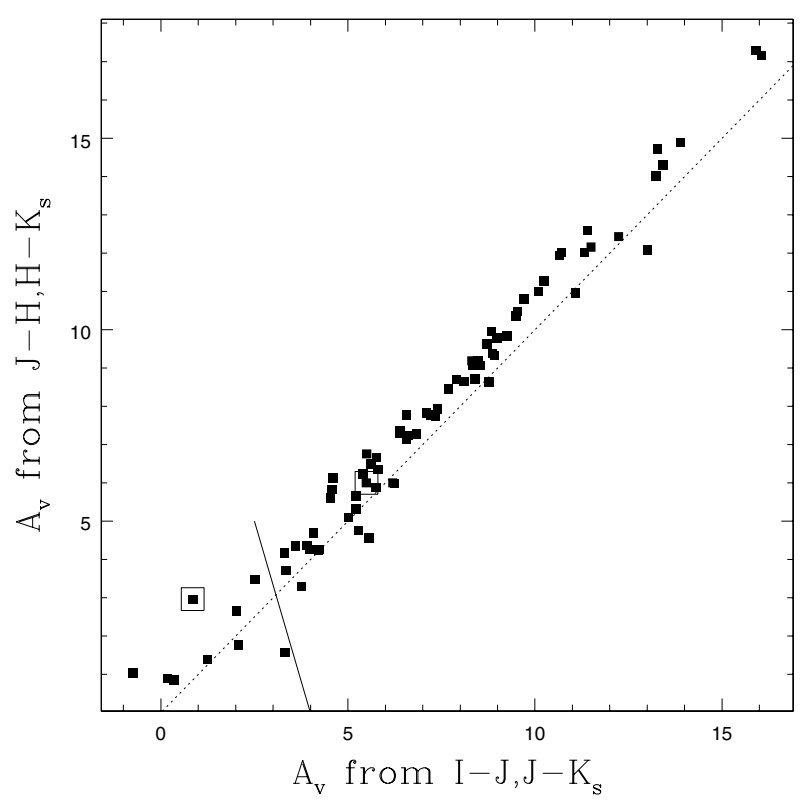

Fig. 5. Comparison of $A_{\mathrm{v}}$ derived by the two different methods indicated by the axis labels and detailed in the text. Both methods yield very similar values. The dotted line denotes equal $A_{\mathrm{v}}$ from each method. We have singled out eight objects with low reddening, lying to the left of the sloping line, plus two more objects highlighted by open squares, for particular discussion.

magnitude for further analysis, we prefer to adopt $A_{\mathrm{v}}$ given by the $J-H$ and $H-K_{\mathrm{s}}$ colours, throughout the remainder of this paper. We note here also, that in addition, we performed similar experiments using a locus in the $I-K_{\mathrm{s}}, J-H$ diagram, as adopted by Luhman et al. (2003) for a study of the Taurus SFR, and found very similar values of $A_{\mathrm{v}}$ in excellent agreement with our preferred methods. Lastly, we are aware that dereddening to dwarf loci may not be correct for NGC 2264 at age 


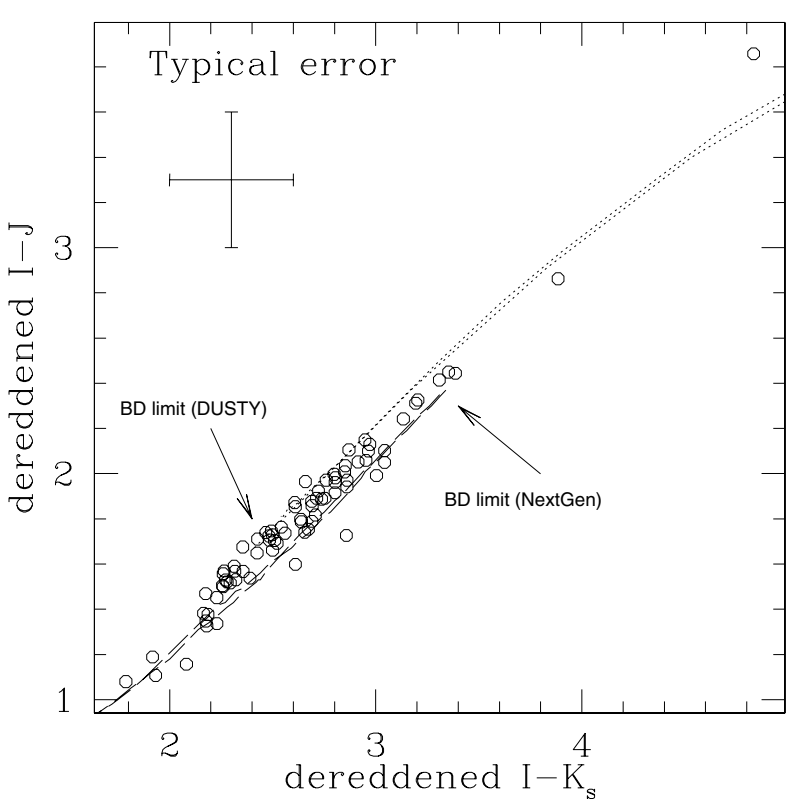

Fig. 6. The $I-J, I-K_{\mathrm{s}}$ diagram, after dereddening. All objects lie close to the theoretical DUSTY and/or NextGen isochrones (dotted and dashed lines, respectively). The substellar limit for suggested by each set of models is indicated; with reference to DUSTY models, many objects are potentially substellar. The typical errors are the same as in Fig. 4.

$3 \mathrm{Myr}$, since young objects have surface gravities intermediate between dwarfs and giants (Greene \& Lada 1996). To illustrate this uncertainty, we have plotted the loci of giant stars of spectral type G0-M 5 from the data of Bessell \& Brett (1988) as dash-dot lines in Figs. 2 and 3. The plotted giant sequence ends at M 5. With reference to Fig. 2, one might assume that it would continue roughly parallel to the dwarf sequence; we do not have data to confirm this. Were it the case, however, one can see from inspection that dereddening to a locus intermediate between dwarfs and giants might yield $A_{\mathrm{v}}$ systematically lower than our estimates, by about one magnitude. Hence our dereddened $I$-magnitudes would be fainter (by about $0.5 \mathrm{mag}$ ) and closer to the observed values, and faint candidate members would therefore be more likely to be substellar with reference to theoretical models. One might also argue that, from Fig. 5, the values of $A_{\mathrm{v}}$ derived from the $J-H, H-K_{\mathrm{s}}$ colour lie above the line of equal $A_{\mathrm{v}}$ with respect to the values from $I-J, J-K_{\mathrm{s}}$, again by about $1 \mathrm{mag}$ Hence by choosing to use $A_{\mathrm{v}}$ derived from $J H K$ colours, we might have overestimated $A_{\mathrm{v}}$ by $\sim 1 \mathrm{mag}$, thus $A_{I}$ by $\sim 0.5 \mathrm{mag}$ In that case, the true values of dereddened $I$ may be fainter by $\sim 0.5 \mathrm{mag}$, and the masses correspondingly lower. For these reasons, our derivation of dereddened magnitudes is conservative.

It is worth noting here that Fig. 4 suggests that we do not observe $K$-band excesses, which generally suggest the presence of an accretion disk. We have re-plotted this diagram in Fig. 6, for dereddened colours. The reader is encouraged to compare Fig. 6 with the Fig. 3 of Caballero et al. (2004), which shows the same plot for a number of $\sigma$ Orionis young brown dwarfs, only one of which shows a clear $K_{\mathrm{s}}$-band excess. For NGC 2264, it is not possible to disentangle the effect of the

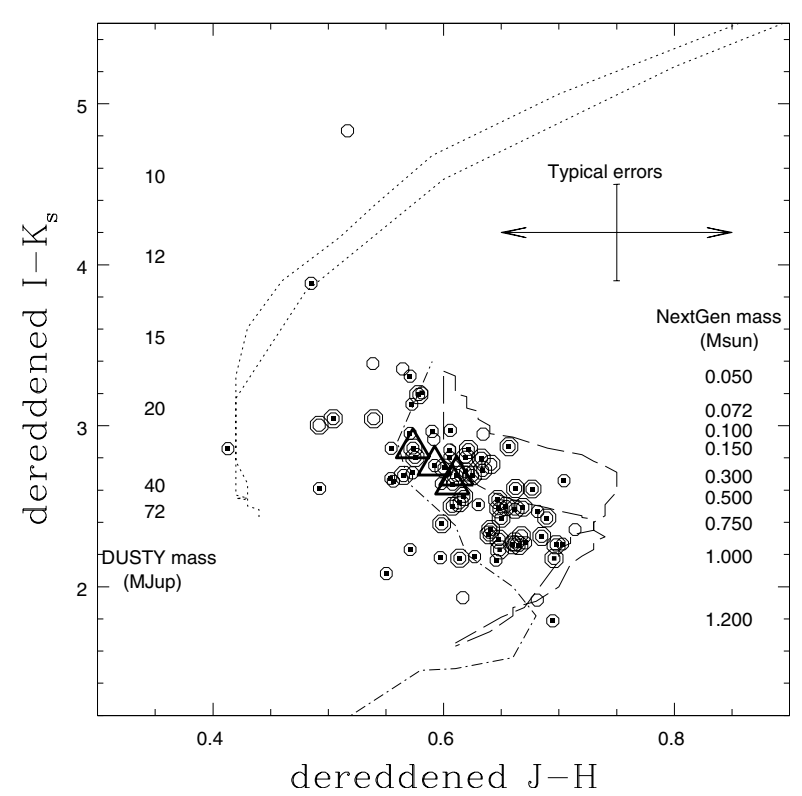

Fig. 7. The sample, plotted in the $I-K_{\mathrm{s}}, J-H$ diagram, after dereddening. The dotted lines represent DUSTY isochrones for ages 1 and $5 \mathrm{Myr}$; the dashed lines are NextGen models for 2 and $5 \mathrm{Myr}$. The dash-dot line is a locus of observed dwarf colours. The errorbar in $J-H$ is large, but mass scales for the mean of each pair of isochrones are derived from the much more diagnostic $I-K_{\mathrm{s}}$ colour, and are shown for DUSTY models (at left) and NextGen (at right). Note that the errorbars do not reflect uncertainties in $A_{\mathrm{v}}$ itself in this or subsequent figures (until Fig. 11, see Sect. 4.3). The whole sample is represented by open circles; points with the best 2MASS photometry (all magnitudes flagged A) are doubly overplotted open symbols. The small filled squares are 65 candidate late-type cluster members (Lamm et al. 2004) recovered in our sample (see text); bold triangles are confirmed variable PMS objects drawn from the same sample by these authors.

intrinsic reddening with the effects of accretion phenomena using near-infrared colours only; observations at longer wavelengths are required.

\section{Discussion}

In this section we will discuss and interpret the nature of our sample by consideration of their dereddened locations in a number of colour-colour and colour-magnitude diagrams, with respect to theoretical DUSTY and NextGen models (Baraffe et al. 2003 and references therein) and other loci derived observationally. Initially, we have treated our values of $A_{\mathrm{v}}$ as exact, deferring a discussion of uncertainties until Sect. 4.3. Hence in Figs. 7 to 10 the errorbars reflect only the observed photometric uncertainties. In Fig. 7 is plotted the $I-K_{\mathrm{s}}$ vs. $J-H$ diagram. The $J-H$ colour is in itself not a diagnostic; all points lie in the range $0.4-0.8$ which is comparable to the likely error in this colour from 2MASS, at least for those objects with poorer quality photometry. However, regarding the $I-K_{\mathrm{s}}$ colour, it can be seen that a large range is covered by our points, spanning likely solar-mass or higher pre-main sequence (PMS) stars (according to the NextGen models) all the way down to around $50 M_{\text {Jup }}$ or even lower, where DUSTY model predictions become appropriate. Our photometry is not 


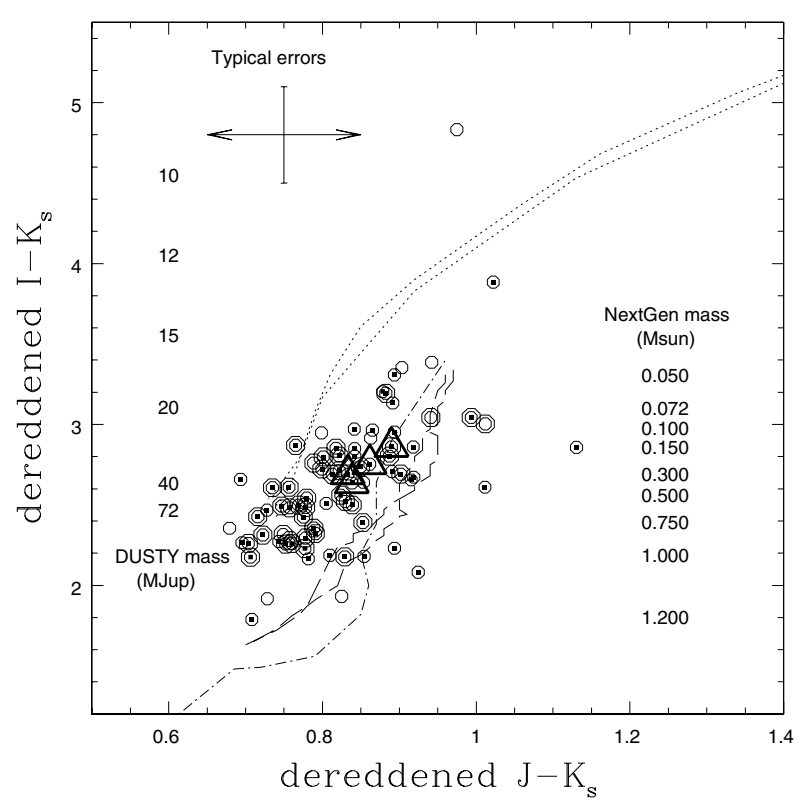

Fig. 8. As Fig. 7, but for dereddened $I-K_{\mathrm{s}}, J-K_{\mathrm{s}}$. Again, mass scales are derived using the $I-K_{\mathrm{s}}$ colour, with typical errobar shown. The error in $J-K_{\mathrm{s}}$ is large, but it is seen that most candidates lie in a space quite consistent with either NextGen or DUSTY models for the cluster (assumed distance modulus 9.4).

accurate enough to distinguish whether a given point lies preferentially near a DUSTY or NextGen model, at any mass where the model predictions overlap. Of course, at the $3 \mathrm{Myr}$ age of NGC 2264, an object at the substellar limit would have spectral type $\sim$ M 6-M 7, above the temperature $(2500-2600 \mathrm{~K}$ or M9/L0) at which dust formation becomes important in the atmospheres of low-mass objects; proven association with a DUSTY isochrone must imply a mass well below this, as the mass scales plotted in Fig. 7 and subsequently show. It is clear from Fig. 7 that almost all points lie at positions extremely consistent with the predictions of NextGen isochrones. This is borne out by a cross-correlation of the 79 objects in our sample with a much larger dataset of 10554 objects in the field of NGC 2264 (Lamm et al. 2004), by which we recover 65 objects, plotted as small filled squares in Fig. 7 and subsequently. However, it should be cautioned that by no means all these objects are confirmed members. Further cross-correlation reveals only four confirmed PMS periodic variables, which are plotted as bold triangles in Figs. 7 and 8 and also as triangles in Fig. 9. We note we have no objects in common with the sample of Rebull et al. (2002), because their limiting magnitude is only $I \sim 17.9$.

It is clear that the vast majority of our faint sample have colours consistent with the NextGen isochrones for NGC 2264. Of course, some fraction could be foreground field stars; the dash-dot line in Fig. 7 represents such a locus of observed dwarf colours given by Leggett (1992) and Kirkpatrick et al. (2000). We do not yet have data to distinguish the cluster and field objects by, for example, gravity-sensitive near-infrared spectroscopy (Lucas et al. 2001). We will return to the topic of foreground field contaminants in more detail in a later section.

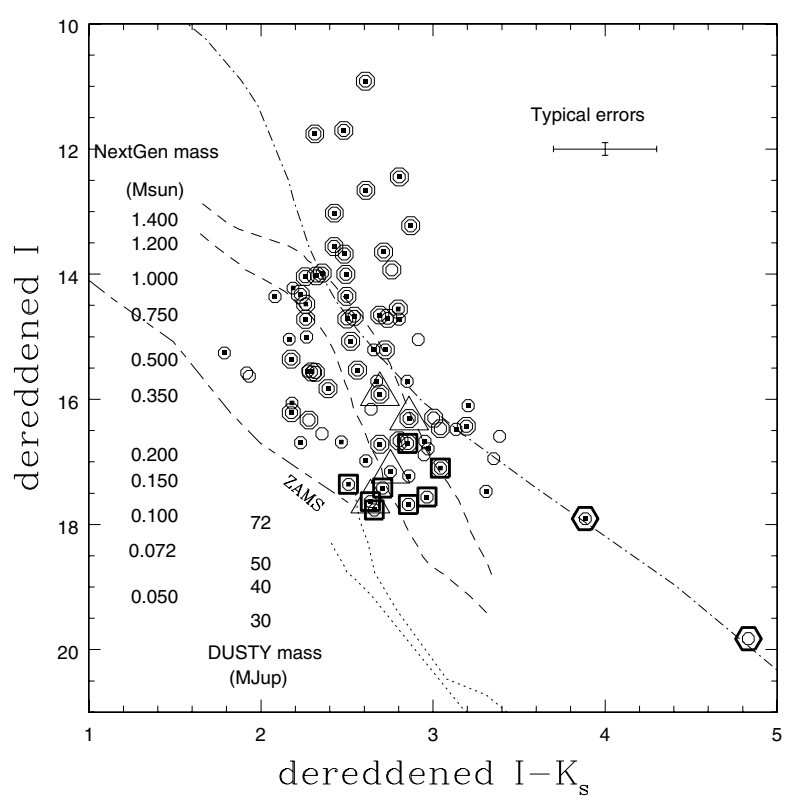

Fig. 9. The dereddened $I, I-K_{\mathrm{s}}$ diagram. The 65 objects we have in common with the sample of Lamm et al. (2004) are plotted as small filled squares; objects with 2MASS photometry flagged AAA are overplotted as open octagons. The errorbar for $I$ is \pm 0.1 mag which is an overestimate for the brighter part of the sample. Note that the errorbars do not take into account uncertainties in $A_{\mathrm{v}}$; i.e. this quantity is treated as exact. Large open triangles indicate the four previously identified NGC 2264 members from Fig. 7. Mass scales are shown for both NextGen and DUSTY models (dashed and dotted lines, respectively). The long/short dashed line is the zero-age main sequence (see text). The dash-dot line is a 5 Gyr field model, chosen for distance modulus 4 ( $63 \mathrm{pc}$ ), chosen to fit the 2 objects with $I-K_{\mathrm{s}}>3.8$ which are discussed in the text. All other objects have locations with respect to the isochrones consistent with them being cluster members with masses above $0.1 M_{\odot}$ (NextGen) but the existence of a few objects very close to the substellar limit is not ruled out by the DUSTY models. The most likely such objects are overplotted by bold squares, and will be discussed in the text. Bold hexagons highlight two further objects whose candidacy will be discussed.

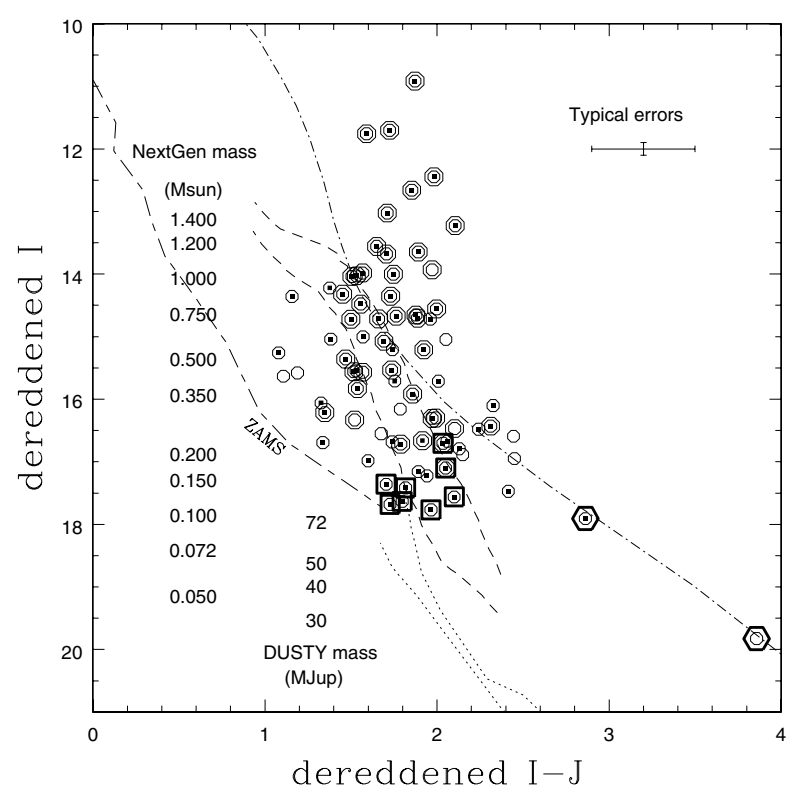

Fig. 10. As for Fig. 9, for dereddened $I, I-J$. 
Table 1. Observed and derived data for NGC 2264 candidates discussed Sect. 4.1 (the first eight) and Sect. 4.2 (last two listed). Columns 7-9 give respectively the $A_{\mathrm{v}}$ derived from near-infrared colours, and the dereddened $I$ and $J$-magnitudes. Uncertainties in $J H K$ are taken to be $\pm 0.1 \mathrm{mag}$ and $\pm 0.3 \mathrm{mag}$ for flags $\mathrm{A}$ and $\mathrm{C}$ respectively, yielding errors in $A_{\mathrm{v}} \pm 1$ mag for flag $\mathrm{A}, \pm 3$ mag for flag C. These errors are carried through to the dereddened magnitudes (see text for details).

\begin{tabular}{lllllllll}
\hline \hline 2MASS designation & $I$ & $J$ & $H$ & $K$ & $A_{\mathrm{v}}$ & der. $I$ & der. $J$ & 2MASS flag \\
\hline 2MASS J06395722+0941011 & 18.09 & 16.00 & 15.27 & 14.87 & 1.39 & $17.4 \pm 0.5$ & $15.6 \pm 0.3$ & AAB \\
2MASS J06400642+0944197 & 18.27 & 16.10 & 15.28 & 15.23 & 1.04 & $17.8 \pm 0.5$ & $15.8 \pm 0.3$ & AAC \\
2MASS J06401053+0939557 & 18.39 & 15.65 & 14.66 & 14.24 & 3.49 & $16.7 \pm 0.5$ & $14.7 \pm 0.3$ & AAA \\
2MASS J06410604+0949232 & 18.00 & 15.71 & 15.03 & 14.70 & 0.89 & $17.6 \pm 0.5$ & $15.5 \pm 0.3$ & AAB \\
2MASS J06402759+0945464 & 18.05 & 16.08 & 15.38 & 15.09 & 0.86 & $17.6 \pm 0.5$ & $15.8 \pm 0.3$ & AAC \\
2MASS J06405674+0938101 & 18.64 & 16.40 & 15.49 & 15.15 & 2.65 & $17.4 \pm 1.0$ & $15.7 \pm 0.6$ & BAC \\
2MASS J06413132+0935120 & 17.95 & 15.55 & 14.86 & 14.26 & 1.77 & $17.1 \pm 0.5$ & $15.1 \pm 0.3$ & AAA \\
2MASS J06401789+0941546 & 18.44 & 16.40 & 15.82 & 15.00 & 1.58 & $17.7 \pm 1.5$ & $16.0 \pm 0.9$ & CBB \\
\hline 2MASS J06404873+0939017 & 21.25 & 16.80 & 15.97 & 15.32 & 2.97 & $19.8 \pm 1.5$ & $16.0 \pm 0.9$ & CCC \\
2MASS J06411281+0945529 & 20.80 & 16.74 & 15.61 & 14.69 & 6.00 & $17.9 \pm 1.5$ & $15.1 \pm 0.9$ & CBB \\
\hline
\end{tabular}

Figure 8 is similar to Fig. 7 except we have plotted the $J-K_{\mathrm{s}}$ colour instead of $H-K_{\mathrm{s}}$. Doing so alleviates slightly the problem of potential large errors in the 2MASS photometry, if only because the $J-K_{\mathrm{s}}$ colour has a larger intrinsic scatter in the sample. Yet, while we are still unable to differentiate between NextGen and DUSTY model predictions here, almost all objects are located consistently with their being NGC 2264 members. Furthermore, a few at the low-mass tip of the NextGen isochrones could be substellar members; clearly for the objects lying near the BD limit of the DUSTY isochrones, further observations are required to disentangle bona fide substellar members from higher mass members and field objects.

For the current study, we need to rely on the absolute magnitudes to yield a truer picture of the status of the objects in our sample. In Fig. 9 we plot $I$ vs. $I-K_{\mathrm{s}}$, in Fig. $10 I$ vs. $I-J$. The zero-age main sequence (ZAMS) is plotted as a long/short dashed line for spectral types earlier than M 5; we have used absolute $V$-magnitudes from Allen (1973) together with observed colours (Kenyon \& Hartmann 1995; Leggett 1992; Kirkpatrick et al. 2000). As should be the case, all points lie above the ZAMS in a region where PMS stars would be located. In no cases are the loci of DUSTY models well populated; with the obvious reason that the $2 \mathrm{MASS}$ photometry utilised here is not sufficiently deep to probe so far below the substellar limit. Nevertheless, these plots demonstrate that our $I, z$-selected sample, as presented here, may well contain some objects at or very close to the substellar limit, with $I$-magnitudes, after dereddening, consistent with both low mass and cluster membership. Such objects lie close to $I=18$ and near the tip of the DUSTY isochrones at $72 M_{\text {Jup }}$, and may either be cluster BD or slightly more massive $\left(\sim 0.1 M_{\odot}\right)$ members according to the NextGen predictions.

\subsection{Likelihood of NGC 2264 membership for selected low $A_{v}$ candidates}

In the following discussion, we concentrate on the eight objects listed in Table 1 and overplotted as bold squares in Figs. 9 and 10. As a group, these candidates are faint $(I \sim 17-18$ after dereddening) and lie close to the BD limit as suggested by DUSTY models, but with higher masses $\sim 0.1-0.15 M_{\odot}$ from the NextGen models. Clearly, all are excellent low-mass NGC 2264 candidates. Only one is a known variable PMS star recovered in the sample of Lamm et al. (2004), as can be seen from Fig. 9. These eight objects are selected to have low $A_{\mathrm{v}}$, lying to the left of the sloping line in Fig. 5. Their observed $I$-magnitudes lie in the range 17.9-18.6 with $I-J \sim 2$; one object lies on the DUSTY isochrone in Fig. 7 with dereddened $I-K_{\mathrm{s}} \sim 2.8$ and $A_{\mathrm{v}} \sim 1.6$, and is a potential $\sim 30 M_{\text {Jup }}$ object by this comparison. Its dereddened $I$-magnitude and $I J K_{\mathrm{s}}$ colours place it at the tip of the 2 Myr DUSTY isochrone in Figs. 9 and 10 yielding a mass at the substellar limit for age $2 \mathrm{Myr}$. Dereddening using $I J K_{\mathrm{s}}$ colours only yields $A_{\mathrm{v}}=3.3$. This object is a good candidate cluster object with potentially substellar mass. Alternative explanations are that, by reference to a 5 Gyr field isochrone (not plotted), it could be a $\sim 0.15 M_{\odot}$ object at $\sim 220$ pc. Such is also suggested by it lying close to the ZAMS with spectral type M 5. Presumably it is insufficiently reddened to be a background giant, behind both NGC 2264 itself and the more distant background dark cloud. If this object is in front of the cloud, its extinction can only be explained by invoking a nearby, hitherto unnoticed, source of reddening, in the foreground of NGC 2264. If a background giant, with $I=17.7$ after dereddening and spectral type M 5, it has $M_{v}=-0.8$ (Allen 1973) and $V-I \sim 3$ (Bessell \& Brett 1988). Its distance would then be an unfeasibly large $200 \mathrm{kpc}$. If instead it is a reddened giant of G0 type $\left(M_{v}=+1.1, V-I \sim 0.8\right)$ then its distance would still be $\sim 30 \mathrm{kpc}$. It is highly unlikely to be a giant at these distances, especially as it is located close to the galactic plane. Since neither the foreground dwarf or background giant hypothesis can explain all the observed characteristics of this object, it is most likely, in our opinion, to be an NGC 2264 member with mass at or close to the BD limit. We note that it has no counterpart in SIMBAD within 9"; the object LBM 2808 (Lamm et al. 2004) is 10" distant and much brighter $(I=16.08)$. Both are clearly visible on the $2 \mathrm{MASS}$ 
$K_{\mathrm{s}}$-band images where the fainter object examined here is a clear point source. Its cc_flag $=000$, so the photometry is uncontaminated; the gal_contam and mp_flg are also both zero; the object is not associated with any extended 2MASS source, nor any solar system object.

As noted, this object has characteristics in common with seven other objects also plotted as bold squares in Figs. 9 and 10; low $A_{\mathrm{v}}$ in the range 0.8-3.5 from the $J-H, H-K_{\mathrm{s}}$ colours, and therefore dereddened $I$-magnitudes suggesting low masses. Taken as a group, it is possible to investigate whether they might be background giants, using similar arguments to the above. If we adopt $I=17$, after dereddening, and $M_{v}=0$ which would be the case for an early K-type giant, then with $V-I=1.2$ (approximately K2III after Bessell $\&$ Brett 1988), we find a distance of $44 \mathrm{kpc}$. The dereddened I-magnitudes of our sample are generally fainter than this (Fig. 10) so derived distances for the eight objects could range up to $\sim 70 \mathrm{kpc}$ (for $I=18$ ). Hence, we consider background contamination extremely unlikely, although it cannot be absolutely ruled out without spectroscopy.

Lastly, for these eight objects we may consider the dereddened $J$-magnitude (Table 1). For a range between 15 and 16, and adopting $M_{v}=0$ and $V-J \sim 2$ (again derived from the colours of an early K-type giant), we find a distance range between 25 and $40 \mathrm{kpc}$. While the distances are feasible, given the moderate reddening and low galactic latitude, it would seem unlikely that these are very distant objects lying beyond both NGC 2264 itself and the background molecular cloud. NGC 2264 membership is much more likely, yielding masses between the substellar limit and $\sim 0.2 M_{\odot}$ from the dereddened $I$ magnitude (Fig. 10) using NextGen isochrones.

\subsection{Two further cases}

Here, we return to examine the cases of the two objects in Figs. 9 and 10 which are marked by bold hexagons. They appear to be preferentially selected by their locations close to DUSTY isochrones in Fig. 7, and are the only two objects with $I-K_{\mathrm{s}}>3.5$; potentially they are cluster members well below the substellar limit. They are very faint, red objects with observed $I \sim 21$, dereddened $I \sim 18$ and 20 and dereddened $J \sim 15$ and 16; their $A_{\mathrm{v}}$ are indicated by the open squares in Fig. 5. Both are also potentially field objects, lying on a $5 \mathrm{Gyr}$ isochrone plotted for $\sim 60 \mathrm{pc}$, chosen to select both objects from their I-magnitudes.

Firstly, we will consider the faintest and reddest object, with $I-K_{\mathrm{s}} \sim 4.8$ (Fig. 7) and dereddened $I \sim 19.8$ (Fig. 9). If a foreground dwarf, its absolute $I$ and $J$-magnitudes suggest it has a mass between 0.075 and $0.08 M_{\odot}$ with respect to the 5 Gyr isochrone; just above the BD limit. From its nearinfrared colours only, we derive $A_{\mathrm{v}}=3$, extremely unlikely for an object so relatively close. $A_{\mathrm{v}}$ derived from the observed $I-J, J-K_{\mathrm{s}}$ colour (Fig. 2) is 0.85 . This lower value is also rather unlikely for an object in close proximity, even near the galactic plane. Nevertheless, it might be that this object is indeed a reddened $\left(A_{\mathrm{v}} \sim 1-3\right)$ dwarf at $\sim 60 \mathrm{pc}$, with a mass just above the brown dwarf limit, and hitherto unnoticed in the galactic plane. There is no counterpart in the SIMBAD database within $10^{\prime \prime}$. Were it an NGC 2264 member, its absolute $I$-magnitude would suggest a mass of $30 M_{\text {Jup }}$; but rather higher from the $J$-magnitude. The current data are unable to tell with certainty whether this object is an NGC 2264 member, or a foreground star. However, it cannot be a background giant; it is too faint, unless it is a very massive star. For example, for a G0 giant with $M_{v}=+1.1$ and $V-I \sim 0.8$, then the distance is $\sim 80 \mathrm{kpc}$; and an intrinsically brighter giant with earlier or later spectral type would be even more remote. Taking all the evidence presented here, we consider that this object is most likely to be an NGC 2264 member with potentially very low mass. However more accurate photometry than provided by 2MASS (this object is flagged " $\mathrm{C}$ " for all magnitudes) is needed.

We now turn to the second of these objects, with $I-K_{\mathrm{s}} \sim$ 3.8 and dereddened $I \sim 18$. Again there is no SIMBAD counterpart within $\sim 8^{\prime \prime}$; the object referred to by Lamm et al. (2004) as LBM 6160 (their Table 4) is within 10", but is much brighter $(I=16.14)$, not a confirmed cluster member according to these authors, and clearly not the same object as discussed below. For our candidate, both methods of calculating $A_{\mathrm{v}}$ yield a value between 5 and 6; it also lies along the field $5 \mathrm{Gyr}, 60 \mathrm{pc}$ isochrone which suggests a mass between 0.08 and $0.09 M_{\odot}$. It is extremely hard to see how a nearby object could be so reddened, and similar arguments to those presented above do not provide a plausible case for the object being a background giant; with G0 giant characteristics, its distance would be $\sim 30 \mathrm{kpc}$. Inspection of Fig. 9 suggests it might be more likely to be a cluster member with $\sim 0.1 M_{\odot}$, by reference to the NextGen isochrones, given the errorbar in $I-K_{\mathrm{s}}$. Indeed, the 2MASS $K_{\mathrm{s}}$-magnitude is flagged "B"; were the object to be in reality fainter at $K_{\mathrm{s}}$, the $I-K_{\mathrm{s}}$ colour would be bluer; closer to the cluster isochrone. It would appear most likely that this object is a hitherto unrecognised, low-mass but probably not substellar, NGC 2264 member.

As a final comment, we note that both these objects have 2MASS flags gal_contam $=0$ and $\mathrm{mp} \_$flg $=0$; thus neither are associated with objects in the extended source catalogue, nor with known solar system objects. The brighter object of the two has a confusion flag associated with the $J$-magnitude; not an uncommon occurence in regions of high stellar density. Presumably this might be because of the proximity on the sky of the Lamm et al. object LBM 6160. Lastly, both appear as faint point sources on inspection of the 2MASS quicklook $K_{\mathrm{s}}$-band images.

In summary, it is apparent that while the membership of these two objects in NGC 2264 remains rather uncertain, and in spite of the fact that they do not lie close to theoretical isochrones for this cluster, simple arguments show that it is highly unlikely that they are either foreground dwarfs, because of their $A_{\mathrm{v}}$, or background giants, because of their low galactic latitude, faintness, and large derived distances. Therefore, they remain excellent candidates for NGC 2264 membership, in which case their $I$-magnitudes place one quite highly reddened object at $\sim 0.1 M_{\odot}$ (NextGen) and suggest the faintest is a cluster member with mass near $30 M_{\text {Jup }}$ (DUSTY). 


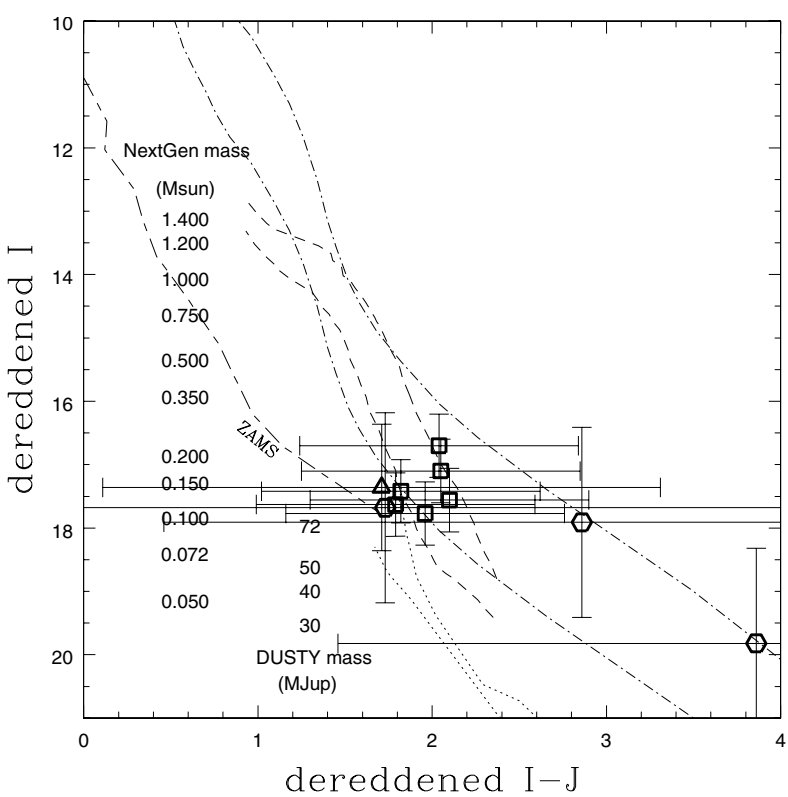

Fig. 11. Dereddened $I, I-J$ diagram illustrating the likely uncertainties on masses once 2MASS photometric errors are propagated through the dereddening process. The bold squares have $J$-mag flag A for which we adopt $\Delta A_{\mathrm{v}}= \pm 1 \mathrm{mag}$, hence $\Delta I$ is $\pm 0.5 \mathrm{mag}, \Delta J \pm$ $0.3 \mathrm{mag}$ and $\Delta I-J \pm 0.8 \mathrm{mag}$ maximum. For the other objects (triangles; $J$ flag B, hexagons; $J$ flag C), we adopt $\Delta A_{\mathrm{v}}= \pm 2$ and 3 mag respectively, with correspondingly larger errorbars in $I$ and $I-J$. Dashdot lines are 5 Gyr NextGen isochrones for distance moduli 4 (as in Fig. 10) and 6. Other isochrones are as Fig. 10.

\subsection{Treatment of errors in dereddened magnitudes}

Before concluding this study, we would like to consider further these ten most probable NGC 2264 members, in particular with a view to investigating contamination by foreground $M$-dwarfs and also how uncertainties in the dereddened magnitudes translate to mass uncertainties. By inspection of the errorbars and magnitude of the reddening vector in Fig. 3, we will adopt uncertainties between \pm 1 and \pm 3 mag in $A_{\mathrm{v}}$ for the objects with 2MASS flags A and C respectively. In general, for these red (faintest at $J$ ) objects, poor accuracy in the 2MASS $J$-magnitude suggests that $J$ is fainter and the $J-H$ colour is larger than given in Fig. 3, and $A_{\mathrm{v}}$ correspondingly greater. Therefore in practice, because $A_{\mathrm{v}}$ is rather more dependent on $J-H$ than $H-K$ (Fig. 3), we have used the $J$-magnitude flag $\mathrm{A}, \mathrm{B}$ or $\mathrm{C}$ to determine $\Delta A_{\mathrm{v}}= \pm 1,2$ or 3 mag, with corresponding $\Delta I$ and $\Delta J$ from the usual relations (Rieke \& Lebofsky 1985), approximated in Fig. 11. Here, we repeat Fig. 10 plotting only the 10 objects discussed in detail above, showing errorbars resulting from the uncertainties in dereddening. It is clear that even for a member of this set at $0.1 M_{\odot}$, with the largest dereddening errors, the final derived mass is nevertheless restricted to a range from $\sim 0.3 M_{\odot}$ (NextGen) down to the substellar limit or below, with the caveat that $A_{\mathrm{v}}$ is more likely to be under- than over-estimated for the faintest objects and masses are therefore likely to be at the upper end of the given range. For objects with the best $J$-band photometry, the errors on the final masses are quite small; the five objects with $J$-flag A (bold squares in Fig. 11) clearly lie

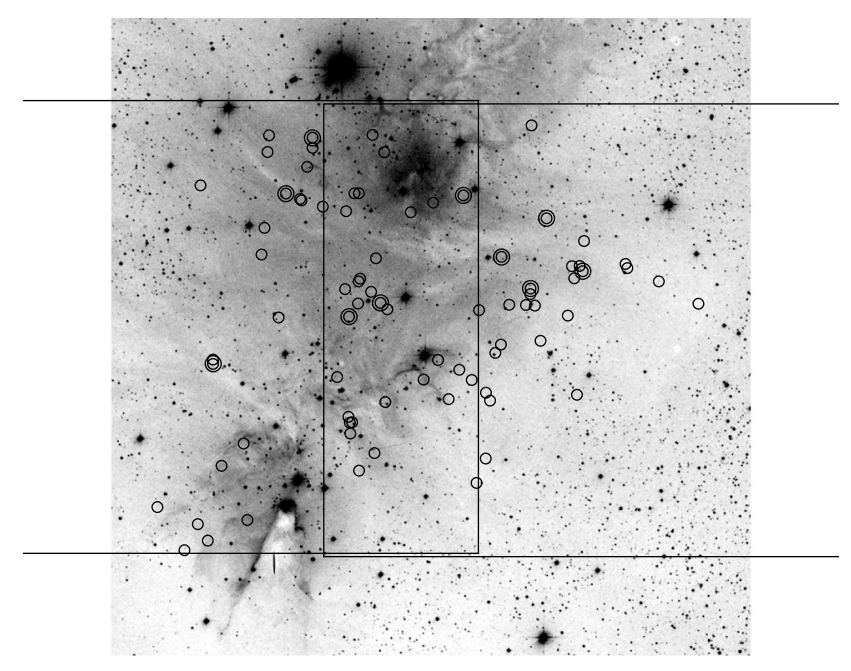

Fig. 12. Spatial location of all 79 candidate objects plotted on the optical ( $R$-band) DSS image which spans $\sim 40^{\prime} \times 40^{\prime}$. The 10 objects discussed in Sects. 4.1 and 4.2 are overplotted as larger $20^{\prime \prime}$ circles. The fields of view of the two $12 \mathrm{~K}$ fields observed are shown, and extend in RA beyond the DSS image. North is up and East to the left.

within $\sim 0.1-0.2 M_{\odot}$ if cluster members. Furthermore, the large error in the dereddened $I-J$ colour for the faintest and reddest candidate does not rule out its being a $\sim 30 M_{\text {Jup }}$ member with reference to the NextGen or DUSTY model isochrones. With regard to contamination by foreground dwarfs, the current data are unable to rule out this possibility, and the main group of candidates have dereddened locations compatible with a 5 Gyr isochrone at distance modulus 6 (Fig. 11). As has already been made clear, spectroscopy is required to identify young NGC 2264 objects by their low surface gravities. It is interesting to note, though, that if a foreground object (at $60 \mathrm{pc}$ ) the faintest object in our sample is itself at the NextGen BD limit. In summary, given the likely uncertainties on the final masses and those inherent in the model predictions, all ten objects remain good candidates for low-mass, NGC 2264 members and possibly cluster brown dwarfs, worthy of further examination.

\section{Concluding remarks}

We have presented our initial findings from a deep, wide-field CFH12K I, $z$-band survey of $\sim 0.6 \mathrm{deg}^{2}$ in NGC 2264 , which is complete to $I \sim 22$, several magnitudes deeper than previously published surveys. The area surveyed is shown in Fig. 12, where the locations of our sample of 79 candidate members are overplotted on the $R$-band DSS image. By cross-correlation with 2MASS, we have been able to accurately deredden all our sample, finding $A_{\mathrm{v}}$ variable, between $\sim 1$ and $\sim 15$, as expected from the clear strong and variable extinction apparent in the image of Fig. 12. Our first important conclusion is that in a study of this type, of any similar region, all near-infrared colours are required to deduce $A_{\mathrm{v}}$ and therefore to be able to compare dereddened photometry with the predictions of model isochrones.

In doing so, we have found that all our sample have photometry consistent with their being NGC 2264 members with 
masses ranging between solar and near the substellar limit. Since all the objects have $I \gtrsim 18$, the most massive candidates are highly reddened, and we only probe close to the BD limit for low $A_{\mathrm{v}}$. We cannot at this stage rule out some contamination by background giants, although significant contamination by such objects is unlikely. We have examined thoroughly the cases of the reddest objects with low $A_{\mathrm{v}}$, and find that all are potentially members with a mass very close to the substellar boundary or below; one may be a member with significantly lower mass. Alternative explanations remain possible, but good arguments can be made to refute them. Infrared spectroscopy is required to finally settle the issue.

Still, we have not yet been able to probe the substellar region with certainty, since the 2MASS survey is not quite deep enough to provide counterparts to all 236 optical candidates; this is not surprising, since at $I=22$ and assuming zero reddening, a substellar member might have $I-K_{\mathrm{s}} \sim 3.5$ (by reference to both observed colours for spectral type $\sim \mathrm{M} 7$ and NextGen isochrones). Hence $K_{\mathrm{s}}$ would be 18.5 , well beyond the reach of $2 \mathrm{MASS}$. Deeper near-infrared photometry is needed; thus NGC 2264 is an excellent target for the next generation of wide-field near-infrared surveys, including the UKIRT/UKIDSS/WFCAM survey (for which NGC 2264 will be observed as part of the Galactic Plane Survey), or CFHT/WIRCAM.

Acknowledgements. T.R.K., E.M. and D.J.J. acknowledge support from the 5th Framework European Union Research Training Network "The Formation and Evolution of Young Stellar Clusters" (RTN11999-00436) and T.R.K. from the French Ministère de la Recherche. E.M. also acknowledges support from The Particle Physics and Astronomy Research Council (PPARC) of the UK. We acknowledge partial financial support from the "Programme National de Physique Stellaire" (PNPS) of CNRS/INSU, France. We thank also the Director of the CFHT for allocation of directors' time to collect part of the data presented here. This publication makes use of data products from the Two Micron All Sky Survey, which is a joint project of the University of Massachusetts and the Infrared Processing and Analysis Centre/California Institute of Technology, funded by the National Aeronautics and Space Administration and the National Science Foundation. This research has made used of the SIMBAD database, operated at CDS, Strasbourg, France. The authors thank the referee, V. J. S. Béjar, for useful comments. Finally, T.R.K. thanks
Jean-Louis Monin for his helpful comments on an earlier version of this work.

\section{References}

Allen, C. W. 1973, Astrophysical Quantities (The Athlone Press) Baraffe, I., Chabrier, G., Barman, T. S., et al. 2003, A\&A, 402, 701 Basri, G. 2000, ARA\&A, 38, 485

Bate, M. R., Bonnell, I. A., \& Bromm, V. 2003, MNRAS, 339, 577

Béjar, V. J. S., Martín, E. L., Zapatero-Osorio, M. R., et al. 2001, ApJ, 556,830

Bertin, E., \& Amouts, S. 1996, A\&AS, 117, 393

Bessell, M. S. 1991, AJ, 101, 622

Bessell, M. S., \& Brett, J. M. 1988, PASP, 100, 1134

Briceño, C., Luhman, K. L., Hartmann, L., et al. 2002, ApJ, 580, 317

Caballero, J. A., Béjar, V. J. S., Rebolo, R., \& Zapatero Osorio, M. R. 2004, A\&A, 424, 857

Chabrier, G. 2002, ApJ, 567, 404

Chabrier, G. 2003, PASP, 115, 763

Chabrier, G., Baraffe, I., Allard, F., \& Hauschildt, P. 2000, ApJ, 542, 464

Cruz, K. L., Reid, I. N., Liebert, J., et al. 2003, AJ, 126, 2421

Greene, T. P., \& Lada, C. J. 1996, AJ, 112, 2184

Kenyon, S. M., \& Hartmann, L. 1995, ApJS, 101, 117

Kirkpatrick, J. D., Reid, I. N., Liebert, J., et al. 2000, AJ, 120, 447

Lamm, M. H., Bailer-Jones, C. A. L., Mundt, R., et al. 2004, A\&A, 417, 557

Landolt, A. U. 1992, AJ, 104, 340

Leggett, S. K. 1992, ApJS, 82, 351

Leggett, S. K., Allard, F., \& Hauschildt, P. H. 1998, ApJ, 509, 836

Lucas, P. W., Roche, P. F., Allard, F., \& Hauschildt, P. H. 2001, MNRAS, 326, 695

Luhman, K. L., Briceno, C., Stauffer, J. R., et al. 2003, ApJ, 590, 348

Martín, E. L., Dougados, C., Magnier, E., et al. 2001, ApJ, 561, L195

Martín, E. L., Delfosse, X., \& Guieu, S. 2004, AJ, 127, 449

Moraux, E., \& Clarke, C. 2005, A\&A, 429, 895

Moraux, E., Bouvier, J., Stauffer, J. R., \& Cuillandre, J.-C. 2003, A\&A, 400, 891

Padoan, P., \& Nordlund, A. 2002, ApJ, 576, 870

Park, B., Sung, H., Bessell, M., \& Kang, Y. 2000, AJ, 120, 894

Rebull, L. M., Makidon, R. B., Strom, S. E., et al. 2002, AJ, 123, 1528

Reipurth, B., \& Clarke, C. 2001, AJ, 122, 432

Rieke, G. K., \& Lebofsky, M. J. 1985, ApJ, 288, 618

Schlegel, D. J., Finkbeiner, D. P., \& Davis, M. 1998, ApJ, 500, 525

Sterzik, M. F., \& Durisen, R. H. 2003, A\&A, 400, 1031

Sung, H., Bessell, M., \& Lee, S.-W. 1997, AJ, 114, 2644 